\title{
FOUR-POINT AFFINE LIE ALGEBRAS
}

\author{
MURRAY BREMNER
}

(Communicated by Roe Goodman)

\begin{abstract}
We consider Lie algebras of the form $\mathfrak{g} \otimes R$ where $\mathfrak{g}$ is a simple complex Lie algebra and $R=\mathbb{C}\left[s, s^{-1},(s-1)^{-1},(s-a)^{-1}\right]$ for $a \in \mathbb{C}-$ $\{0,1\}$. After showing that $R$ is isomorphic to a quadratic extension of the ring $\mathbb{C}\left[t, t^{-1}\right]$ of Laurent polynomials, we prove that $g \otimes R$ is a quasi-graded Lie algebra with a triangular decomposition. We determine the universal central extension of $\mathfrak{g} \otimes R$ and show that the cocycles defining it are closely related to ultraspherical (Gegenbauer) polynomials.
\end{abstract}

\section{INTRODUCTION}

Let $\mathfrak{g}$ be a simple finite-dimensional complex Lie algebra. By the loop algebra of $\mathfrak{g}$ we mean the infinite-dimensional complex Lie algebra $\mathscr{G}=\mathfrak{g} \otimes \mathbb{C}\left[t, t^{-1}\right]$ with commutation relations $[x \otimes f, y \otimes g]=[x y] \otimes f g$, where $x, y \in \mathfrak{g}$ and $f, g \in \mathbb{C}\left[t, t^{-1}\right]$. The universal central extension $\hat{\mathscr{G}}$ of the loop algebra has a 1-dimensional kernel $\mathbb{C} \omega$; we call $\hat{\mathscr{G}}$ an untwisted affine Kac-Moody Lie algebra. Since the canonical central element $\omega$ of $\hat{\mathscr{G}}$ acts by nonzero scalar multiplication on many interesting representations (e.g. the integrable irreducible highest weight modules), the central extension is essential for the representation theory. The structure and representation theory of affine KacMoody Lie algebras has been studied in great detail by mathematicians and physicists during the last 25 years; two comprehensive expositions of this subject are [Kac] and [MP].

The Laurent polynomial ring $\mathbb{C}\left[t, t^{-1}\right]$ is the ring of rational functions on the Riemann sphere $\mathbb{C} \cup\{\infty\}$ with poles allowed only in $\{\infty, 0\}$. This geometric point of view suggests a natural generalization of the loop algebra construction. Instead of the sphere with two punctures, one can consider any complex algebraic curve $\Sigma$ of genus $g$ with a fixed subset $P$ of $n$ distinct points. We let $R$ denote the ring of meromorphic functions on $\Sigma$ with poles allowed only in $P$, and we form the Lie algebra $\mathscr{G}=\mathfrak{g} \otimes R$. Using a result from [Kas], we can then determine the universal central extension $\dot{\mathscr{G}}$ of this Lie algebra (with a view towards its representation theory).

Received by the editors July 1, 1993 and, in revised form, November 1, 1993.

1991 Mathematics Subject Classification. Primary 17B05, 17B65; Secondary 17B67, 33C45, $81 \mathrm{R} 10,14 \mathrm{H} 99$.

The author thanks the Natural Sciences and Engineering Research Council of Canada for financial support. 
In the case of genus 0 , the Lie algebras obtained by this procedure (called $n$ point affine Lie algebras) were introduced in [B]. The case of genus 1, with two specified points, has been considered in [Sh1,Sh2]. These papers were inspired by the fundamental work [KN] of Krichever and Novikov, which studied the Lie algebras of vector fields on Riemann surfaces of arbitrary genus with poles allowed only at two specified points, and by the work [Sc] which studied the $n$-point generalization of these Lie algebras.

The $n$-point affine Lie algebras have recently appeared in the work of Kazhdan and Lusztig [KLu1,2], inspired by conformal field theory, on tensor structures on categories of modules over affine Kac-Moody Lie algebras.

For helpful comments on an earlier version of this paper I thank the members of the Lie Theory Seminar at the University of Saskatchewan, Roe Goodman, and the referee.

\section{THE 4-POINT RING}

Let $a_{1}, \ldots, a_{4}$ be any four distinct points on the Riemann sphere $\mathbb{C} \cup\{\infty\}$ with coordinate $s$, and let $R$ be the ring of rational functions with poles allowed only in $\left\{a_{1}, \ldots, a_{4}\right\}$. Thus $R$ is a subring of the field $\mathbb{C}(s)$ of rational functions; since the automorphism group $P G L_{2}(\mathbb{C})$ of $\mathbb{C}(s)$ is (simply) 3-transitive, we may assume that $a_{1}=\infty, a_{2}=0$ and $a_{3}=1$. Writing $a_{4}=a$, we define the 4-point ring to be $R=R_{a}=\mathbb{C}\left[s, s^{-1},(s-1)^{-1},(s-a)^{-1}\right]$ with $a \in \mathbb{C}-\{0,1\}$. We also consider the ring $S=S_{b}=\mathbb{C}\left[t, t^{-1}, u\right]$ where $u^{2}=t^{2}-2 b t+1$ with $b \in \mathbb{C}-\{ \pm 1\}$.

Proposition 1.1. We have $R_{a} \simeq S_{b}$ where $b=(a+1) /(a-1) \in \mathbb{C}-\{ \pm 1\}$. Conversely we have $S_{b} \simeq R_{a}$ where $a=(b+1) /(b-1) \in \mathbb{C}-\{0,1\}$.

Proof. Since $\mathbb{C}[s]$ is a subring of $R_{a}$, inverting $s-1$ and $s-a$ is equivaient to inverting $(s-1)(s-a)$. Since $s^{-1} \in R_{a}$, inverting $(s-1)(s-a)$ is equivalent to inverting $s^{-1}(s-1)(s-a)$. Hence $R_{a}=\mathbb{C}\left[s, s^{-1}, t_{1}^{-1}\right]$, where $t_{1}=s-(a+1)+$ $a s^{-1}$; we also set $u_{1}=s-a s^{-1}$. Since $a \neq 0$, we have $\mathbb{C}\left[s, s^{-1}\right]=\mathbb{C}\left[t_{1}, u_{1}\right]$, and so $R_{a}=\mathbb{C}\left[t_{1}, t_{1}^{-1}, u_{1}\right]$. We easily check that $u_{1}^{2}=t_{1}^{2}+2(a+1) t_{1}+(a-1)^{2}$, and so we set $t=-t_{1} /(a-1), u=-u_{1} /(a-1)$ to obtain $u^{2}=t^{2}-2 b t+1$ where $b=(a+1) /(a-1)$. This shows that the correspondence

$$
t=\frac{-1}{a-1}\left(s-(a+1)+a s^{-1}\right), \quad u=\frac{-1}{a-1}\left(s-a s^{-1}\right)
$$

is an isomorphism between $S_{b}$ and $R_{a}$.

For the converse, we can solve for $a$ in terms of $b$, and $s, s^{-1}$ in terms of $t, u$. The same result may be obtained as follows. Completing the square in $u^{2}=t^{2}-2 b t+1$ and rearranging gives $\left(u^{2}-(t-b)^{2}\right) /\left(1-b^{2}\right)=1$. We now set

$$
s=\frac{1}{1-b}(u+t-b), \quad s^{-1}=\frac{1}{1+b}(u-t+b) ;
$$

we will show that this correspondence is an isomorphism between $R_{a}$ and $S_{b}$. Since $\mathbb{C}[t, u]=\mathbb{C}\left[s, s^{-1}\right]$, we have $S_{b}=\mathbb{C}\left[t, t^{-1}, u\right]=\mathbb{C}\left[s, s^{-1}, t^{-1}\right]=$ $\mathbb{C}\left[s, s^{-1},(s t)^{-1}\right]$. Solving for $t$ in terms of $s$ and $s^{-1}$ we get

$$
s t=\frac{1}{2}(1-b)(s-1)(s-(b+1) /(b-1)) .
$$

Therefore $S_{b}=R_{a}$ with $a=(b+1) /(b-1)$. 
This result shows that the 4-point ring is a quadratic extension of the ring of Laurent polynomials. The geometric reason for this isomorphism is as follows. Starting with the sphere with two punctures we can blow up each puncture to two points, thereby obtaining the sphere with four punctures. This gives a surjective map from the sphere with four punctures to the sphere with two punctures. Now looking instead at the rings of functions, we conclude that the ring of functions on the sphere with four punctures is a module of rank 2 over the subring $\mathbb{C}\left[t, t^{-1}\right]$ of functions on the sphere with two punctures.

Since $P G L_{2}(\mathbb{C})$ is 3-transitive, an automorphism of $R_{a}$ is uniquely determined by the permutation it induces on the set $\{\infty, 0,1, a\}$. Thus the automorphism group of $R_{a}$ is always isomorphic to a subgroup of the symmetric group on four letters.

Proposition 1.2. For any $a \in \mathbb{C}-\{0,1\}$, the ring $R_{a}$ has a 4-group of automorphisms $\{1, p, q, p q\}$ given by

$$
1(s)=s, \quad p(s)=\frac{s-a}{s-1}, \quad q(s)=\frac{a(s-1)}{s-a}, \quad p q(s)=\frac{a}{s} .
$$

As permutations of the set $\{\infty, 0,1, a\}$ we have $p=(\infty 1)(0 a), q=(\infty a)(01)$, $p q=(\infty 0)(1 a)$. In terms of $t$ and $u$ these automorphisms take the forms

$$
\begin{gathered}
p(t)=t^{-1}, p(u)=t^{-1} u, \quad q(t)=t^{-1}, q(u)=-t^{-1} u, \\
p q(t)=t, p q(u)=-u .
\end{gathered}
$$

Proof. The first two assertions are easy calculations. For the third, we use the equation $p\left(s^{-1}\right)=(s-1) /(s-a)$ together with the formulas for $t, u$ in terms of $s, s^{-1}$ in the previous proposition.

It seems appropriate to mention at this point (although we will not need the result) that the above four automorphisms give the full automorphism group of $R_{a}$ except in the cases $a \in\left\{-1, \frac{1}{2}, 2\right\}$ - here we obtain a group isomorphic to the dihedral group of order 8 - and $a \in\left\{\frac{1}{2} \pm \frac{1}{2} \sqrt{-3}\right\}$ - here we obtain a group isomorphic to the alternating group of order 12 . This result can be obtained by determining, for each permutation $\pi$ of $\{\infty, 0,1, a\}$, the values of $a$ for which an element of $P G L_{2}(\mathbb{C})$ exists having the same action as $\pi$ on the four points.

\section{ThE QUASI-GRADING AND TRIANGULAR DECOMPOSITION OF $\mathfrak{g} \otimes R$}

From now on we identify $R=R_{a}$ with $S_{b}$, so $R$ has a basis consisting of $t^{i}, t^{i} u$ for $i \in \mathbb{Z}$. There is a $\mathbb{Z} / 2$-grading on $R=R^{0} \oplus R^{1}$ defined by $R^{0}=\mathbb{C}\left[t, t^{-1}\right], R^{1}=\mathbb{C}\left[t, t^{-1}\right] u$; here $R^{i}$ is the $(-1)^{i}$-eigenspace of $p q$.

We write $\mathscr{G}$ for $g \otimes R$, and call this the (untwisted) 4-point loop algebra. The $\mathbb{Z} / 2$-grading on $R$ induces the structure of a $\mathbb{Z} / 2$-graded Lie algebra on $\mathscr{G}$ by setting $\mathscr{G}^{0}=\mathfrak{g} \otimes R^{0}, \mathscr{G}^{1}=\mathfrak{g} \otimes R^{1}$. The advantage of realizing the 4-point ring $R$ as a $\mathbb{Z} / 2$-graded extension of $\mathbb{C}\left[t, t^{-1}\right]$ is that this allows us to display $\mathscr{G}$ as a quasi-graded Lie algebra with a triangular decomposition, which is linearly isomorphic to the direct sum of the ordinary loop algebra $\mathfrak{g} \otimes \mathbb{C}\left[t, t^{-1}\right]$ and a copy of its adjoint representation $\mathfrak{g} \otimes \mathbb{C}\left[t, t^{-1}\right] u$.

We recall the definition of a quasi-graded algebra from $[\mathrm{KN}]$. Let $I$ be a subgroup of the additive group $\mathbb{Q}$, and let $\mathscr{A}$ be an algebra. For each $i \in I$ 
let $\mathscr{A}_{i}$ be a subspace of $\mathscr{A}$, and assume that $\mathscr{A}=\bigoplus_{i \in I} \mathscr{A}_{i}$. Let $l \in I, l \geq 0$. If we have

$$
x \in \mathscr{A}_{i}, y \in \mathscr{A}_{j} \Rightarrow x y \in \bigoplus_{|k-(i+j)| \leq l} \mathscr{A}_{k},
$$

then we call $\mathscr{A}$ an l-quasi-graded algebra. If $l=0$, then we recover the usual definition of a graded algebra. If $x \in \mathscr{A}_{i}, x \neq 0$, then (by a slight abuse of terminology) we say that $x$ is homogeneous of degree $i$ and we write $\operatorname{deg} x=i$.

By a weak triangular decomposition of the Lie algebra $\mathscr{G}$ we mean a triple $\left(\mathscr{H}, \mathscr{G}_{+}, \sigma\right)$ where

(1) $\mathscr{H}$ and $\mathscr{G}_{+}$are subalgebras of $\mathscr{G}$,

(2) $\mathscr{H}$ is Abelian and $\left[\mathscr{H} \mathscr{G}_{+}\right] \subseteq \mathscr{G}_{+}$,

(3) $\sigma$ is an anti-automorphism of $\mathscr{G}$ of order 2 which fixes $\mathscr{H}$ pointwise, and

(4) $\mathscr{G}$ is the direct sum of the subspaces $\mathscr{H}, \mathscr{G}_{+}$and $\sigma\left(\mathscr{G}_{+}\right)$.

We write $\mathscr{G}_{-}=\sigma\left(\mathscr{G}_{+}\right)$so we have $\mathscr{G}=\mathscr{G}_{-} \oplus \mathscr{H} \oplus \mathscr{G}_{+}$. From (2) and (3) it follows that $\left[\mathscr{K} \mathscr{G}_{-}\right] \subseteq \mathscr{G}_{-}$. For the definition of a (strict) triangular decomposition see [MP].

Theorem 2.1. The 4-point loop algebra $\mathscr{G}$ is a 1-quasi-graded Lie algebra with a weak triangular decomposition.

Proof. We make $R$ into a 1-quasi-graded (commutative associative) algebra by taking $I=\frac{1}{2} \mathbb{Z}$ and setting $\operatorname{deg} t^{i}=i, \operatorname{deg} t^{i} u=i+\frac{1}{2}$. We then make $\mathscr{G}$ into a 1-quasi-graded Lie algebra with the same $I$ by setting $\operatorname{deg}\left(x \otimes t^{i}\right)=i$ and $\operatorname{deg}\left(x \otimes t^{i} u\right)=i+\frac{1}{2}$ for any $x \in \mathfrak{g}, x \neq 0$.

Let $\mathfrak{g}=\mathfrak{g}_{-} \oplus \mathfrak{h} \oplus \mathfrak{g}_{+}$be the usual triangular decomposition: $\mathfrak{h}$ is a Cartan subalgebra, and $\mathfrak{g}_{+}$(resp. $\mathfrak{g}_{-}$) is the sum of the positive (resp. negative) root spaces with respect to $\mathfrak{h}$. We decompose $R$ as the direct sum of the three subspaces $\mathbb{C}, R_{+}=\mathbb{C}(1+u) \oplus \mathbb{C}[t, u] t$ and $R_{-}=p\left(R_{+}\right)=\mathbb{C}\left(1+t^{-1} u\right) \oplus$ $\mathbb{C}\left[t^{-1}, u\right] t^{-1}$. Note that $(1+u)^{2}=2(1+u)-2 b t+t^{2}$, so $R_{+}$and $R_{-}$are in fact subrings. Combining these decompositions we set $\mathscr{H}=\mathfrak{h} \otimes \mathbb{C}, \mathscr{G}_{+}=$ $\left(\mathfrak{g}_{+} \otimes \mathbb{C}\right) \oplus\left(\mathfrak{g} \otimes R_{+}\right)$and $\mathscr{G}_{-}=\left(\mathfrak{g}_{-} \otimes \mathbb{C}\right) \oplus\left(\mathfrak{g} \otimes R_{-}\right)$. Letting $e$ denote the negative of the Chevalley involution of $\mathfrak{g}$, we define an anti-automorphism $\sigma$ of $\mathscr{G}=\mathfrak{g} \otimes R$ by $\sigma=e \otimes p$. Then $\left(\mathscr{H}, \mathscr{G}_{+}, \sigma\right)$ is a weak triangular decomposition of $\mathscr{G}$.

This triangular decomposition of $\mathscr{G}$ is inhomogeneous with respect to the $\mathbb{Z} / 2$-grading of $\mathscr{G}$ since it involves the elements $1+u$ and $1+t^{-1} u$. Note also that $\mathscr{G}_{+}$(resp. $\mathscr{G}_{-}$) is not the sum of the homogeneous subspaces of $\mathscr{G}$ with positive (resp. negative) degree.

We can also write $\mathscr{G}$ as the sum of the two subalgebras $\mathfrak{g} \otimes \mathbb{C}[t, u]$ and $\mathfrak{g} \otimes$ $\mathbb{C}\left[t^{-1}, t^{-1} u\right]$ which intersect in the subalgebra $\mathfrak{g} \otimes \mathbb{C}$. The proof of Proposition 1.1 shows that $\mathbb{C}[t, u] \simeq \mathbb{C}\left[s, s^{-1}\right]$; applying $p$ we see that $\mathbb{C}\left[t^{-1}, t^{-1} u\right] \simeq$ $\mathbb{C}\left[s_{1}, s_{1}^{-1}\right]$ for $s_{1}=(s-a) /(s-1)$.

\section{The Universal CENTRAL EXTENSION OF $\mathfrak{g} \otimes R$}

By Kassel's theorem [Kas], we know that for any commutative associative $\mathbb{C}$-algebra $R$, the universal central extension of $\mathfrak{g} \otimes R$ is linearly isomorphic to $(\mathfrak{g} \otimes R) \oplus \Omega_{R}^{1} / d R$, where $\Omega_{R}^{1} / d R$ is the space of Kähler differentials of $R$ 
modulo exact differentials. Remarkably the kernel $\Omega_{R}^{1} / d R$ of the extension does not depend on the Lie algebra $\mathfrak{g}$, so determining the central extension is essentially a problem in commutative algebra.

We define $\Omega_{R}^{1} / d R$ as the cyclic homology group $H C_{1}(R)$, following [KL]. Let $F=R \otimes R$ be the left $R$-module with action $f(g \otimes h)=f g \otimes h$ for $f, g, h \in R$. Let $K$ be the submodule generated by the elements $1 \otimes f g-$ $f \otimes g-g \otimes f$. Let $\Omega_{R}^{1}=F / K$ be the quotient module; this is the module of differentials of $R$. We denote the element $f \otimes g+K$ of $\Omega_{R}^{1}$ by $f d g$. We define a map $d: R \rightarrow \Omega_{R}^{1}$ by $d(f)=d f=1 \otimes f+K$. The elements of the subspace $d R=d(R)$ are exact differentials. We denote the coset of $f d g$ modulo $d R$ by $\overline{f d g}$. The commutation relations for $(\mathfrak{g} \otimes R) \oplus \Omega_{R}^{1} / d R$ are

$$
[x \otimes f, y \otimes g]=[x y] \otimes f g+(x, y) \overline{f d g}, \quad[x \otimes f, \omega]=0,
$$

where $x, y \in \mathfrak{g}, f, g \in R$, and $\omega \in \Omega_{R}^{1} / d R$; here $(x, y)$ denotes the Killing form on $\mathfrak{g}$.

In our case we have $R=S_{b}$ and $\mathscr{G}=\mathfrak{g} \otimes R$. We set $\hat{\mathscr{G}}=\mathscr{G} \oplus \Omega_{R}^{1} / d R$. To make the commutation relations for $\hat{\mathscr{G}}$ explicit we need to compute a basis for $\Omega_{R}^{1} / d R$ and the skew-symmetric mapping $R \times R \rightarrow \Omega_{R}^{1} / d R$ given by the composition

$$
R \times R \rightarrow R \otimes R \rightarrow(R \otimes R) / K=\Omega_{R}^{1} \rightarrow \Omega_{R}^{1} / d R .
$$

All of these objects have a $\mathbb{Z} / 2$-grading induced by that on $R$. As usual we will call elements of degree 0 even and elements of degree 1 odd.

The elements $t^{i} \otimes t^{j}, t^{i} \otimes t^{j} u, t^{i} u \otimes t^{j}$ and $t^{i} u \otimes t^{j} u$ form a basis of $R \otimes R$.

Lemma 3.1. $\Omega_{R}^{1}$ is spanned by the differentials $t^{i} d t, t^{i} u d t$ and $t^{i} d u$ for $i \in \mathbb{Z}$. Proof. We have to show that any basis element of $R \otimes R$ is congruent modulo $K$ to an element in the span of $t^{i} \otimes t, t^{i} \otimes u$, and $t^{i} u \otimes t$.

Clearly $f \otimes g \equiv 0(\bmod K)$ is equivalent to $f d g=0$ in $\Omega_{R}^{1} / d R$. One easily shows by induction that $d\left(t^{j}\right)=j t^{j-1} d t$ and that $d\left(t^{j} u\right)=j t^{j-1} u d t+t^{j} d u$. Since $K$ is a submodule of $R \otimes R$, we can multiply each of the above equations by $t^{i}$ or $t^{i} u$. This shows that any basis element of $R \otimes R$ is equal to an element in the span of $t^{i} d t, t^{i} d u, t^{i} u d t$ and $t^{i} u d u$.

To complete the proof, we use the fact that $u d u=\frac{1}{2} d\left(u^{2}\right)$. Since $u^{2}=$ $t^{2}-2 b t+1$, we find that $u d u=t d t-b d t$. We now multiply this equation by $t^{i}$ to get the result.

Lemma 3.2. $\Omega_{R}^{1}$ is spanned by the differentials $t^{i} d t, t^{i} u d t$ for $i \in \mathbb{Z}$, together with $d u$ and $t d u$.

Proof. We know that $\frac{1}{2} u d\left(u^{2}\right)=u^{2} d u$. Since $u^{2}=t^{2}-2 b t+1$, we find that

$$
t u d t-b u d t-t^{2} d u+2 b t d u-d u=0 .
$$

We multiply this equation by $t^{i}$ to get

$$
t^{i+1} u d t-b t^{i} u d t-t^{i+2} d u+2 b t^{i+1} d u-t^{i} d u=0 .
$$

For $i \geq 0$ this shows that $t^{i+2} d u$ is equal to a linear combination of $t^{i+1} d u$, $t^{i} d u$ and elements of the form $t^{j} u d t$. For $i \leq-1$ it shows that $t^{i} d u$ is equal to a linear combination of $t^{i+1} d u, t^{i+2} d u$ and elements of the form $t^{j} u d t$. From this we easily show by induction that any element of the form $t^{i} d u$ is 
equal to a linear combination of $d u, t d u$ and elements of the form $t^{j} u d t$. Now Lemma 3.1 completes the proof.

Theorem 3.3. A basis of $\Omega_{R}^{1} / d R$ is $\left\{\overline{t^{-1} d t}, \overline{t^{-2} u d t}, \overline{t^{-1} u d t}\right\}$.

Proof. The $\mathbb{Z} / 2$-grading of $\Omega_{R}^{1}$ and $d R$ gives $\Omega_{R}^{1} / d R=\left(\Omega_{R}^{1}\right)^{0} / d\left(R^{0}\right) \oplus$ $\left(\Omega_{R}^{1}\right)^{1} / d\left(R^{1}\right)$.

We first consider the even subspace. We have $d\left(t^{i}\right)=i t^{i-1} d t$ for all $i \in \mathbb{Z}$. From this we immediately see that $t^{i-1} d t \equiv 0(\bmod d R)$ for $i \neq 0$. Therefore $\left(\Omega_{R}^{1}\right)^{0} / d\left(R^{0}\right)$ is spanned by $\overline{t^{-1} d t}$.

Next we consider the odd subspace. We have $d\left(t^{i} u\right)=i t^{i-1} u d t+t^{i} d u$, and so $t^{i} d u \equiv-i t^{i-1} u d t(\bmod d R)$. The odd subspace of $\Omega_{R}^{1}$ is spanned by $t^{i} u d t$ together with $d u$ and $t d u$. Clearly $d u \equiv 0(\bmod d R)$ and $t d u \equiv-u d t(\bmod$ $d R$ ). Thus we only need to consider the elements $t^{i} u d t$. We will show that modulo $d R$ all these elements are congruent to a linear combination of $t^{-2} u d t$ and $t^{-1} u d t$.

First suppose that $i \leq-3$. Then $t^{i} u d t \equiv-\frac{1}{i+1} t^{i+1} d u(\bmod d R)$. By formula (3.2.1) we know that $t^{i+1} d u$ is a linear combination of $t^{i+2} d u, t^{i+3} d u$, $t^{i+1} u d t$ and $t^{i+2} u d t$. Modulo $d R$ we have $t^{i+2} d u \equiv-(i+2) t^{i+1} u d t$ and $t^{i+3} d u \equiv-(i+3) t^{i+2} u d t$. Hence $t^{i} u d t$ is congruent to a linear combination $t^{i+1} u d t$ and $t^{i+2} u d t$. From this we see by induction that $t^{i} u d t$ for $i \leq-3$ is congruent modulo $d R$ to a linear combination of $t^{-2} u d t$ and $t^{-1} u d t$.

Next suppose that $i \geq 0$. As before $t^{i} u d t \equiv-\frac{1}{i+1} t^{i+1} d u(\bmod d R)$, and using (3.2.1) again we know that $t^{i+1} d u$ is a linear combination of $t^{i-1} d u, t^{i} d u$, $t^{i-1} u d t$ and $t^{i} u d t$. Since the coefficient of $t^{i} u d t$ in this linear combination is 1 , we can solve for $t^{i} u d t$, showing that it is congruent to a linear combination of $t^{i-1} d u, t^{i} d u$ and $t^{i-1} u d t$. Since $t^{i-1} d u \equiv-(i-1) t^{i-2} u d t(\bmod d R)$ and $t^{i} d u \equiv-i t^{i-1} u d t(\bmod d R)$ we conclude that $t^{i} u d t$ is congruent to a linear combination of $t^{i-1} u d t$ and $t^{i-2} u d t$. As before we see by induction that $t^{i} u d t$ for $i \geq 0$ is congruent modulo $d R$ to a linear combination of $t^{-2} u d t$ and $t^{-1} u d t$.

We know by a result of [B] that $\operatorname{dim} \Omega_{R}^{1} / d R=3$, and so these three spanning elements are linearly independent. Other references for this dimension calculation, which includes the computation of higher homology groups, are [CHG, LL].

We define $\omega_{0}=\overline{t^{-1} d t}, \omega_{-}=\overline{t^{-2} u d t}$, and $\omega_{+}=\overline{t^{-1} u d t}$. We then easily check that

$$
\begin{aligned}
p\left(\omega_{0}\right) & =-\omega_{0}, & p\left(\omega_{-}\right) & =-\omega_{+}, & p\left(\omega_{+}\right) & =-\omega_{-}, \\
q\left(\omega_{0}\right) & =-\omega_{0}, & q\left(\omega_{-}\right) & =\omega_{+}, & q\left(\omega_{+}\right) & =\omega_{-}, \\
p q\left(\omega_{0}\right) & =\omega_{0}, & p q\left(\omega_{-}\right) & =-\omega_{-}, & p q\left(\omega_{+}\right) & =-\omega_{+} .
\end{aligned}
$$

To make the commutation relations for $\hat{\mathscr{G}}$ precise we need to compute $\overline{f d g}$ for any basis elements $f, g \in R$. We deal separately with the two cases $f g$ even and $f g$ odd. Note that $\overline{f d g}$ is always the linear combination of $\left\{\omega_{0}, \omega_{+}, \omega_{-}\right\}$which gives the congruence class of $f d g$ modulo $d R$.

Proposition 3.4 (The even case). For $i, j \in \mathbb{Z}$ we have

$$
\overline{t^{i} d\left(t^{j}\right)}= \begin{cases}j \omega_{0} & \text { for } i+j=0, \\ 0 & \text { for } i+j \neq 0\end{cases}
$$


For $i, j \in \mathbb{Z}+\frac{1}{2}$ we have

$$
\overline{t^{i-\frac{1}{2}} u d\left(t^{j-\frac{1}{2}} u\right)}= \begin{cases}\left(j+\frac{1}{2}\right) \omega_{0} & \text { for } i+j=-1 \\ -2 j b \omega_{0} & \text { for } i+j=0 \\ \left(j-\frac{1}{2}\right) \omega_{0} & \text { for } i+j=1 \\ 0 & \text { for }|i+j| \geq 2\end{cases}
$$

Proof. The first equation follows from the relation $t^{i} d\left(t^{j}\right)=j t^{i+j-1} d t$. The relation

$$
t^{i-\frac{1}{2}} u d\left(t^{j-\frac{1}{2}} u\right)=\left(j+\frac{1}{2}\right) t^{i+j} d t-2 j b t^{i+j-1} d t+\left(j-\frac{1}{2}\right) t^{i+j-2} d t
$$

implies the second congruence.

The odd case is more complicated. By skew-symmetry we only need to consider the elements $t^{i-\frac{1}{2}} u \otimes t^{j}$ for $i \in \mathbb{Z}+\frac{1}{2}, j \in \mathbb{Z}$. We recall the definition of the ultraspherical (Gegenbauer) polynomials $P_{k}^{(\lambda)}(x)$. These may be defined in two ways [Sz]: either by the generating function

$$
\sum_{n=0}^{\infty} P_{n}^{(\lambda)}(x) w^{n}=\left(1-2 x w+w^{2}\right)^{-\lambda}
$$

or by the initial values $P_{0}^{(\lambda)}(x)=1$ and $P_{1}^{(\lambda)}(x)=2 \lambda x$ and the recursion formula

$$
n P_{n}^{(\lambda)}(x)=2(n+\lambda-1) x P_{n-1}^{(\lambda)}(x)-(n+2 \lambda-2) P_{n-2}^{(\lambda)}(x) .
$$

We only need the case $\lambda=-\frac{1}{2}$, so we write $P_{k}(b)=P_{k}^{(-1 / 2)}(b)$. We also define

$$
Q_{k}(b)=-\frac{P_{k+2}(b)}{b^{2}-1} \text {. }
$$

If $b= \pm 1$, then $\left(1-2 b t+t^{2}\right)^{1 / 2}= \pm(1 \pm t)$, so $b^{2}-1$ divides $P_{k+2}(b)$ for $k \geq 0$; hence $Q_{k}(b)$ is a polynomial. The first eight polynomials in this sequence are

$$
\begin{gathered}
Q_{0}=\frac{1}{2}, \quad Q_{1}=\frac{1}{2} b, \quad Q_{2}=\frac{1}{8}\left(5 b^{2}-1\right), \quad Q_{3}=\frac{1}{8}\left(7 b^{3}-3 b\right), \\
Q_{4}=\frac{1}{16}\left(21 b^{4}-14 b^{2}+1\right), \quad Q_{5}=\frac{1}{16}\left(33 b^{5}-30 b^{3}+5 b\right), \\
Q_{6}=\frac{1}{128}\left(429 b^{6}-495 b^{4}+135 b^{2}-5\right), \\
Q_{7}=\frac{1}{128}\left(715 b^{7}-1001 b^{5}+385 b^{3}-35 b\right) .
\end{gathered}
$$

Proposition 3.5 (The odd case). For $i \in \mathbb{Z}+\frac{1}{2}, j \in \mathbb{Z}$, we have

$$
\overline{t^{i-\frac{1}{2}} u d\left(t^{j}\right)}= \begin{cases}j Q_{i+j-\frac{3}{2}}(b)\left(b \omega_{+}+\omega_{-}\right) & \text {for } i+j \geq \frac{3}{2}, \\ j \omega_{ \pm} & \text {for } i+j= \pm \frac{1}{2}, \\ j Q_{-i-j-\frac{3}{2}}(b)\left(\omega_{+}+b \omega_{-}\right) & \text {for } i+j \leq-\frac{3}{2} .\end{cases}
$$

Proof. We know that $t^{i-\frac{1}{2}} u d\left(t^{j}\right)=j t^{i+j-\frac{3}{2}} u d t$. The cases $i+j= \pm \frac{1}{2}$ then follow from the definition of $\omega_{ \pm}$. The case $i+j \leq-\frac{3}{2}$ follows from the case $i+1 \geq \frac{3}{2}$ by applying the automorphism $p$. Thus it suffices to consider the case $i+j \geq \frac{3}{2}$. For this we need only to determine the congruence class of $t^{k} u d t$ $(\bmod d R)$ for $k \in \mathbb{Z}, k \geq 0$. 
We start by making explicit the recursion in the proof of Theorem 3.3. Expanding $d\left(t^{k+1} u\right)$ we find that

$$
(k+1) t^{k} u d t \equiv-t^{k+1} d u \quad(\bmod d R) .
$$

Rewriting formula (3.2.1) we get

$$
t^{k+1} d u=t^{k} u d t-b t^{k-1} u d t+2 b t^{k} d u-t^{k-1} d u .
$$

Combining these two results gives

$$
(k+2) t^{k} u d t \equiv b t^{k-1} u d t-2 b t^{k} d u+t^{k-1} d u \quad(\bmod d R) .
$$

Using formula (3.5.1) again we find

$$
(k+2) t^{k} u d t \equiv b t^{k-1} u d t+2 k b t^{k-1} u d t-(k-1) t^{k-2} u d t \quad(\bmod d R) .
$$

Therefore

$$
(k+2) \overline{t^{k} u d t}=(2 k+1) b \overline{t^{k-1} u d t}-(k-1) \overline{t^{k-2} u d t} .
$$

For $0 \leq k \leq 1$ this gives

$$
\overline{u d t}=\frac{1}{2}\left(b \omega_{+}+\omega_{-}\right), \quad \overline{t u d t}=\frac{1}{2} b\left(b \omega_{+}+\omega_{-}\right) .
$$

From this it follows that for all $k \geq 0$ we have $\overline{t^{k} u d t}=f_{k}(b)\left(b \omega_{+}+\omega_{-}\right)$, where $f_{k}(b)$ is a polynomial in $b$. We have the recursion formula

$$
f_{0}=\frac{1}{2}, \quad f_{1}=\frac{1}{2} b, \quad(k+2) f_{k}=(2 k+1) b f_{k-1}-(k-1) f_{k-2} .
$$

Setting $g_{k}(b)=-\left(b^{2}-1\right) f_{k}(b)$ and writing $n=k+2$ we have the equivalent formula

$g_{2}=-\frac{1}{2}\left(b^{2}-1\right), \quad g_{3}=-\frac{1}{2} b\left(b^{2}-1\right), \quad n g_{n}=2\left(n--\frac{3}{2}\right) b g_{n-1}-(n-3) g_{n-2}$.

Therefore, by the formula for $P^{(\lambda)}(x)$ given above, we see that $g_{n}(b)=$ $P_{n}^{(-1 / 2)}(b)$ and so $f_{k}(b)=Q_{k}(b)$.

We can now give explicit commutation relations for $\hat{\mathscr{G}}$.

Theorem 3.6. The 4-point affine Lie algebra $\hat{\mathscr{G}}$ has a $\mathbb{Z} / 2$-grading in which

$$
\hat{\mathscr{G}}^{0}=\mathfrak{g} \otimes \mathbb{C}\left[t, t^{-1}\right] \oplus \mathbb{C} \omega_{0}, \quad \hat{\mathscr{G}}^{1}=\mathfrak{g} \otimes \mathbb{C}\left[t, t^{-1}\right] u \oplus \mathbb{C} \omega_{+} \oplus \mathbb{C} \omega_{-} .
$$

A spanning subset of $\hat{\mathscr{G}}$ consists of the elements $x \otimes t^{i}$ and $x \otimes t^{j-\frac{1}{2}} u$ where $x \in \mathfrak{g}, \quad i \in \mathbb{Z}, j \in \mathbb{Z}+\frac{1}{2}$, together with the central elements $\omega_{0}, \omega_{+}$, and $\omega_{-}$. The even subalgebra $\hat{\mathscr{G}}^{0}$ is an untwisted affine Kac-Moody Lie algebra with commutation relations

$$
\left[x \otimes t^{i}, y \otimes t^{j}\right]=[x y] \otimes t^{i+j}+\delta_{i+j, 0}(x, y) j \omega_{0} .
$$

The commutator of tw'o elements of $\hat{\mathscr{G}}^{1}$ lies in $\hat{\mathscr{G}}^{0}$ :

$\left[x \otimes t^{i-\frac{1}{2}} u, y \otimes t^{j-\frac{1}{2}} u\right]$

$$
=[x y] \otimes\left(t^{i+j-1}-2 b t^{i+j}+t^{i+j+1}\right)+(x, y) \begin{cases}-2 j b \omega_{0} & \text { for } i+j=0, \\ \frac{1}{2}(j-i) \omega_{0} & \text { for }|i+j|=1, \\ 0 & \text { for }|i+j| \geq 2 .\end{cases}
$$


The odd subspace $\hat{\mathscr{G}}^{1}$ is a $\hat{\mathscr{G}}^{0}$-module with relations

$$
\begin{aligned}
& {\left[x \otimes t^{i-\frac{1}{2}} u, y \otimes t^{j}\right]} \\
& =[x y] \otimes t^{i+j-\frac{1}{2}} u+(x, y) \begin{cases}j Q_{i+j-\frac{3}{2}}(b)\left(b \omega_{+}+\omega_{-}\right) & \text {for } i+j \geq \frac{3}{2}, \\
j \omega_{ \pm} & \text {for } i+j= \pm \frac{1}{2}, \\
j Q_{-i-j-\frac{3}{2}}(b)\left(\omega_{+}+b \omega_{-}\right) & \text {for } i+j \leq-\frac{3}{2} .\end{cases}
\end{aligned}
$$

Proof. Since the $\mathbb{Z} / 2$-grading on $R$ induces a $\mathbb{Z} / 2$-grading on both $\mathscr{G}$ and $\Omega_{R}^{1} / d R$, the 4-point affine Lie algebra $\hat{\mathscr{G}}$ also has a $\mathbb{Z} / 2$-grading. The other assertions are clear.

Note that $\hat{\mathscr{G}}$ is not a 1-quasi-graded Lie algebra; to get a 1-quasi-graded Lie algebra we must factor out the central ideal spanned by the odd central elements $\omega_{-}$and $\omega_{+}$. The reason for this is that the construction of $\Omega_{R}^{1} / d R$ respects the grading on the subring $\mathbb{C}\left[t, t^{-1}\right]$ of $R$ but not the 1-quasi-grading of $R$. Thus it makes sense to set $\operatorname{deg} \omega_{0}=0$, but there is no reasonable choice of values for $\operatorname{deg} \omega_{ \pm}$.

\section{REFERENCES}

[B] M. Bremner, Generalized affine Kac-Moody Lie algebras over localizations of the polynomial ring in one variable, Canad. Math. Bull. 37 (1994), 21-28.

[CHG] The Buenos Aires Cyclic Homology Group, Cyclic homology of monogenic algebras, preprint.

[Kac] V. G. Kac, Infinite dimensional Lie algebras, 3rd edition, Cambridge Univ. Press, Cambridge, 1990.

[Kas] C. Kassel, Kähler differentials and coverings of complex simple Lie algebras extended over a commutative algebra, J. Pure Appl. Algebra 34 (1984), 265-275.

[KL] C. Kassel and J.-L. Loday, Extensions centrales d'algèbres de Lie, Ann. Inst. Fourier (Grenoble) 32 (1982), 119-142.

[KLu1] D. Kazhdan and G. Lusztig, Affine Lie algebras and quantum groups, Internat. Math. Res. Notes 2 (1991), 21-29.

[KLu2] _ , Tensor structures arising from affine Lie algebras, I and II, preprint.

[KN] I. M. Krichever and S. P. Novikov, Algebras of Virasoro type, Riemann surfaces and structures of the theory of solitons, Funct. Anal. Appl. 21 (1987), 126-142.

[LL] M. Larsen and A. Lindenstrauss, Cyclic homology of Dedekind domains, $K$-Theory 6 (1992), 301-334.

[MP] R. Moody and A. Pianzola, Lie algebras with triangular decomposition, Wiley, New York (to appear).

[Sc] M. Schlichenmaier, Krichever-Novikov algebras for more than two points, Lett. Math. Phys. 19 (1990), 151-165.

[Sh1] O. Sheinman, Elliptic affine Lie algebras, Funct. Anal. Appl. 24 (1990), 210-219.

[Sh2] Highest weight modules over certain quasigraded Lie algebras on elliptic curves, Funct. Anal. Appl. 26 (1992), 203-208.

[Sz] G. Szegö, Orthogonal polynomials, 4th ed., Amer. Math. Soc., Providence, RI, 1975.

\footnotetext{
Department of Mathematics, University of Toronto, Toronto, Ontario, Canada M5S $1 \mathrm{~A} 1$

Current address: Department of Mathematics and Statistics, McLean Hall, Room 142, University of Saskatchewan, 106 Wiggins Road, Saskatoon, Saskatchewan, Canada S7N 5E6

E-mail address: bremner@math.usask.ca
} 